\title{
Dynamic Wireless Information and Power Transfer Optimization Scheme for Nano-Empowered Vehicular Networks
}

\author{
Li Feng, Amjad Ali, Muddesar Iqbal, Farman Ali, Imran Raza, Muhammad Hameed Saddiqui, Muhammad \\ Shafiq, and Syed Asad Hussain
}

\begin{abstract}
In this paper, we investigate the wireless power transfer and energy-efficiency (EE) optimization problem for nano-centric vehicular networks operating over the terahertz band. The inbody nano-sensors harvest energy from a power station via radio-frequency signal and then use the harvested energy to transmit data to the sink node. By considering the properties of terahertz band (i.e., sensitivity to distance and frequency over the communication path), we adopt the Brownian motion model to develop a time-variant terahertz channel model and to describe the mobility of the nano-sensors. Thus, based on the channel model and energy resources, we further develop a long-term EE optimization problem. The EE optimization is further converted into a series of energy-efficient resource allocation problems over the time slots via equivalent transformation method. The resource allocation problem for each timeslot, which is formulated as a mixed integer nonlinear programming (MINLP), is solved based on the particle swarm optimization (PSO) method. In addition, a dynamic PSO-based EE optimization (DPEEO) algorithm is developed to obtain the sub-optimal solution for the $\mathrm{EE}$ optimization problem. By exploiting the special structure of the reformulated problem, an improved DPEEO algorithm, is presented which can handle the problem's constraints quite well, decreases the research space, and greatly reduces the length of the convergence time. Simulation results validate the theoretical analysis of our system.
\end{abstract}

Key words: nano-communications vehicular networks, wireless power transfer, energy-efficiency optimization, terahertz band

\section{INTRODUCTION}

In the present era of advance technology, vehicles are getting more efficient, sophisticated, faster than the past. Moreover, with the significant advancements in nanotechnology, nanotechnology-empowered sensors over terahertz band are

L. Feng is with the School of Computer Science and Communication Engineering, Jiangsu University, 301 Xuefu Road, Zhenjiang, 212013, Jiangsu, China. (Email: fenglixidian@gmail).

A. Ali, I.Raza, and S.A. Hussain are with the Department of Computer Science COMSATS University Islamabad, Lahore Campus, Pakistan. (Email: amjad.ali@cuilahore.edu.pk

M. Iqbal is with the Division of Computer Science and Informatics, London South Bank University, London SE1 OAA, UK.

M. H. Saddiqui is with the College of Computer and Information Sciences, Jouf University, Sakaka, Saudi Arabia

F. Ali is with the Department of Software, Sejong University, Seoul, South Korea

M. Shafiq is with the Department of Information and Communication Engineering, Yeungnam University, Gyeongsan 38541, South Korea.

This project is funded by MSCA-RISE-Marie-Sklodowska-Curie Research and Innovation Staff Exchange (RISE) scheme under the project SONNET (Self-OrganizatioN towards reduced cost and eNergy per bit for future Emerging radio Technologies) with grant agreement no. H2020-MSCA-RISE2016-73454 expected to bring a wide range of applications in vehicular networks from the comfort to entertainment [1]. From $0.1 \mathrm{THz}$ - $10 \mathrm{THz}$ wireless spectrum is reserved for terahertz communication which has great capability to support extremely high bandwidths communication. However, some constraints, such as frequency selective path-loss and noise may lead fluctuations in terahertz channel capacity [2]. Besides, due to their physical dimensions, nano-sensors implanted in vehicles are always limited by the requirement for a battery. Therefore, wireless power transfer (WPT) technology over the air interface is introduced as a viable solution to solve the problem of battery and network lifetime [3].

Energy efficiency (EE) optimization has become a vital design objective in green vehicular communications. Despite the numerous advantages of nano-centric vehicular communications over terahertz band, there are still many challenges being faced in energy efficient transmissions using nano-sensors which requires innovative techniques to be develop. Thus, due to the fact that a nano-sensor's energy resource will change with its energy harvesting and consumption processes, first we need to build a dynamic equation to describe the time-varying energy resources. Secondly, since the channel character varies with the frequency and distance on the communication path, a comprehensive mathematical model is required to portray the time-variant channels of the terahertz band for vehicular networks. Thirdly, considering the time-varying energy resources and channels, a dynamic EE optimization problem shall be addressed to improve the vehicular network performance. Besides, owing to the complex channel property of the terahertz band, the problem built is non-convex, which is difficult to solve. Some efficient mathematic methods are needed to obtain the problem's sub-optimal solutions. Therefore, in this paper, we propose a dynamic wireless information and power transfer scheme to maximize the long-term EE by jointly considering the time-varying energy resources and the channels for nanotechnology-empowered vehicular networks using terahertz band.

In our paper, we employ Brownian motion to describe the mobility of a nano-sensor and build a time-variant terahertz channel model, due to the fact that terahertz communications is sensitive to distance and frequency over the communication path. Meanwhile, based on the power transfer and energy consumption processes, we build a stochastic equation to model the dynamic energy resource of the nano-sensor. Considering nano-sensors' dynamic channel information and energy 
resources, we develop a long-term optimization to maximize the EE in the vehicular networks. To solve the non-convex optimization problem, we convert it into a series of energyefficient resource allocation problems based on the equivalent transformation method. The resource allocation problem for each time slot is further solved based on particle swarm optimization (PSO) method [4]. Some major contributions of this paper are given below.

- We employ wireless power transfer technology, and build time-varying channel and energy models of the nanotechnology-empowered sensor implanted in vehicular networks over the terahertz band.

- We build a long-term optimization for EE under energy resources and the time-varying channel model for the nano-sensors.

- A dynamic PSO-based EE optimization (DPEEO) algorithm is proposed to obtain the sub-optimal solution to the optimization problem. Based on the special structure of the reformulated problem, we further present an improved DPEEO algorithm, which can handle the problem's constraints quite well, decreases the research space, and greatly reduces the length of convergence time.

Our remaining paper's organization is as follows. Section II presents the state-of-the-art. Section III and IV discusses the system model and the problem formulation, respectively. Section $\mathrm{V}$ proposes the two dynamic EE optimization algorithms. Finally, simulation results and conclusion is presented in Section VI, and VII, respectively.

\section{RELATED WORKS}

Studies [5-6] discussed the resource allocation problems for improving the energy in wireless networks. A EE optimization algorithm is proposed that jointly consider the power allocation and sub-carrier for improving the energy efficiency in multiuser wireless networks. Similarly, in [6], an optimization objective function is developed to deal with energy efficiency under the queue-aware multimedia heterogeneous cloud radio access networks. However, the proposed solutions may not be directly adopted nano sensors based vehicular network. When it comes to the nanoscale scene, there are some resource allocation strategies for nano-sensors. Thus, in order to improve the overall network transmission rate, a channel selection scheme for nanoscale devices is introduced in [7] that is based on terahertz band. Similarly, in order to improve a channel capacity, a power allocation technique is also proposed for terahertz band in [8]. To ensure the improved data transmissions, a energy-aware routing protocol is developed in [9]. Works [7-9] introduced some resource allocation methods; however, they do not consider nanotechnology for communication. Furthermore, some other dynamic properties, such as the change in distance on the communication path and the time-varying chemical composition also not considered, which may cause a variant channel in the terahertz band and strongly affects the achievable data rates.

By considering the time-varying chemical composition properties, a frequency-hopping technique for adjusting the rate of frequency switching for nano-sensors is introduced in
[10]. For modeling the mobility of nano-sensors in nanocommunications networks and to deal with the mobile nanosensors, a random walk-based model is proposed in [11]. Both [10-11] do not considered the terahertz band for nanocommunications networks. Because of the technological limitations, the conventional energy harvesting techniques, such as underwater turbulence, solar energy, and wind power cannot be directly adopted in nano-communications networks. Therefore, some novel schemes are required to provide energy to nanosensors. Thus, energy harvesting is considered as one of the vital solution to solve the energy-related problems in wireless devices [12]. In [3], authors proposed a energy harvesting scheme for piezoelectric nano-generators. In [14], authors introduced a complex energy harvesting scheme for wireless nano-networks operating over terahertz band. However, the energy harvesting models are mostly not much reliable and controllable, or do not capture the properties of wireless communications in nano-communications networks.

Recently, performance maximization in wireless networks is usually formulated as a convex optimization problem, where conventional solving techniques are adopted [15-16]. However, practical data transmission systems always introduce nonconvex utility functions, and a very few works addressed the non-convex optimization problems. In front of the nonconvex optimization including integer and continuous variables, in order to obtain the sub-optimal solution to energyefficient resource optimization a continuous convex approximation method is introduced in [17]. To deal with the power consumption optimization problem under wireless networks, a branch-and-bound scheme is used in [18]. However, owing to the channel's complex property for the terahertz band, it is still difficult to find a convex problem to approximate the proposed original non-convex problem. Then, the methods presented above cannot be applied well to the EE optimization problem of nano-communications networks in the terahertz band. Thus, there is a need to develop intelligent technique to achieve the optimum solution for the nanotechnologyempowered vehicular networks. Particle swarm optimization (PSO) technique belongs to the evolutionary solution's family that is mainly based on the imitation of the foraging behavior for a flock of birds learning and grouping the best experiences [19]. To find the optimal deployment problem solution in a non-convex region, a discrete PSO scheme is introduced in [20]. PSO is computationally more efficient and has the advantages of the ease of implementation and good convergence. In our previous work [38], we proposed a resource allocation scheme for time-variant channel under nano-coomunication networks. In the scheme, we addressed the power allocation and channel selection optimization problems and solve them by employing the Lyapunov optimization. Finally, a algorithm called "CoRA" is developed.

\section{SYSTEM MODEL}

\section{A. Network Model}

In the considered nano-centric vehicular communications system, there are $N$ nano-sensors, one sink node, and one power station. The nano-sensors, $\mathcal{N}$, travel via Brownian motion with 


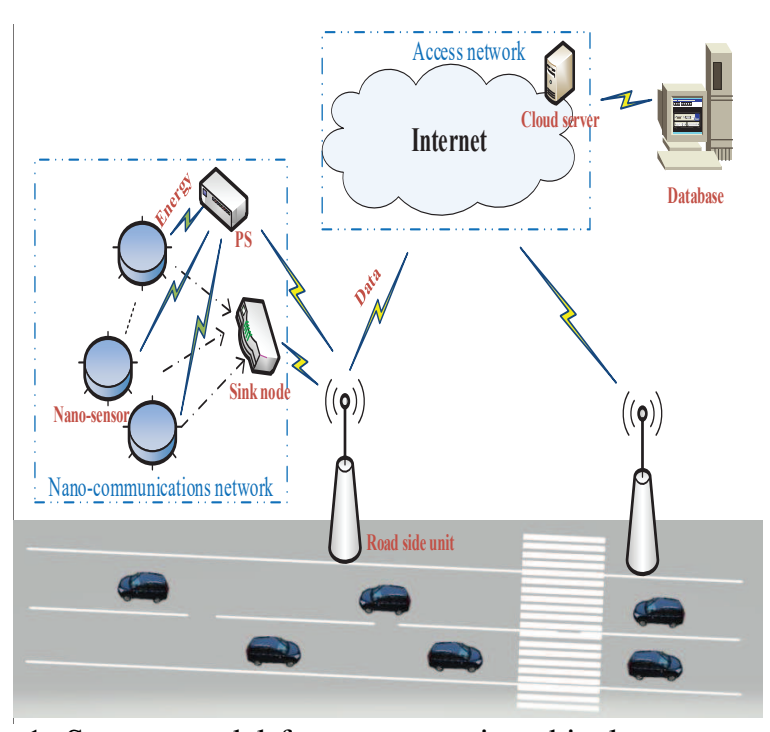

Fig. 1: System model for nano-centric vehicular communications networks operating over the terahertz band.

drift. These nano-sensors sense some vital statistic, such as trajectory of enemy vehicles and transmit the sensed data to the sink node over a single-hop communication as demonstrated in Fig. 1. Besides, the sink node is capable of performing complex tasks, and it also forward the collected packets to higher layers, e.g., a road side units (RSUs). Then, the nanosensors can access the Internet via the RSU. To execute data transmission, each nano-sensor is comprised on a communication block and a power block along with the relevant storage units and transmission process. Due to the limitation of nanosensor's small size, both power block and communication block are finite [21].

To ensure the system operates smoothly, each nano-sensor (which has no embedded power supply available) needs to harvest energy to power itself. Here, we introduce wireless power transfer (WPT) technology into the considered network scenario, and the power station (PS), which acts as a wireless power source, can transmit the wireless power to each nanosensor via radio frequency signals (e.g., WiFi, ultra-wide band and so on). The nano-sensors operate over the terahertz band and total bandwidth of the terahertz band is divided into $M$ narrow frequencies or channel with equal-width $\Delta f$. Thus, multiple nano-sensors can operate over different subfrequency bands or channels. Moreover, due to operating over orthogonal channel, we assume no interference exists among nano-sensors. $\mathcal{M}$, denotes the channel set, a nano-sensor $n$ can transmit its data over a discrete frequency $f_{m}, m \in \mathcal{M}$. We considered that the channel characteristics of data transmission for nano-sensors may vary with their motions and similarly, energy resources may also vary over the time during power transfer and data transmission processes. Thus, we considered a time-slotted environment where a time is slotted into equal intervals indexed by $t, t=\{0,1,2, \cdots\}$. The network state (power block information and channel property) for each nano-sensor is assumed to be constant for the duration of one time slot, but potentially change at slot boundaries. In a specific time slot, a harvest-then-transmit protocol is employed for data transmission in the network. The protocol is divided

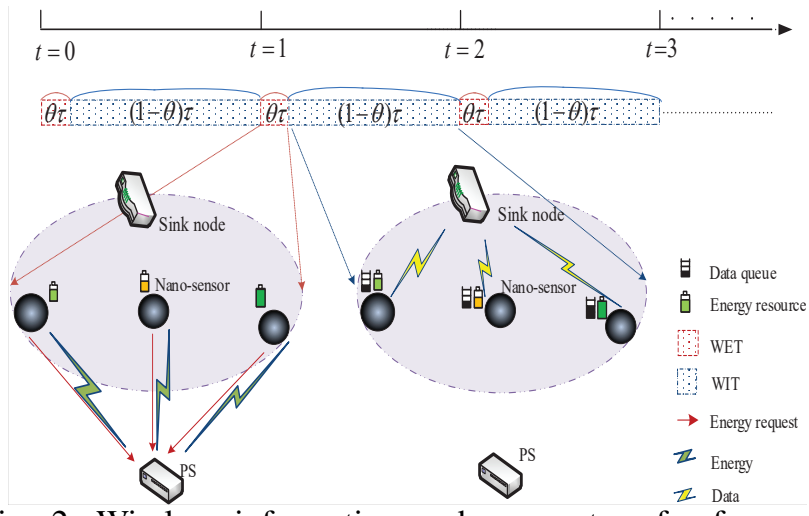

Fig. 2: Wireless information and power transfer for nanocentric vehicular communications networks.

into two parts: (a) Wireless energy transfer (WET), i.e., the PS provides wireless power for the nano-sensors via RF signals, and the nano-sensors store the harvested energy in their limited power blocks. (b) Wireless information transfer (WIT), i.e., the nano-sensors employ the received power resources to forward the data packets to the sink node using terahertz bands. Specifically, for a $\theta$ duration of time slot $t$ (the WET stage), each nano-sensor decides the amount of energy required based on its power block information at the beginning and then sends a power request to the PS. When the PS receives the power requests, it transfers power resources to the nanosensors via RF signal. At the rest of the time (the WIT stage), the nano-sensors may utilize the received power and forward the data packets to the sink node via terahertz bands. When the network state changes and time slot $t+1$ comes, new power transfer and data transmission periods (among the nano-sensors, sink node and PS) start, as shown in Fig. 2. Additionally, due to the fact that the energy transfer and data forwarding processes are executed at different times and at different frequencies ${ }^{2}$, no interference exists between the nanosensors and the PS.

\section{B. Motion Model}

In this paper, $\vec{d}_{n}^{\mathbb{E}, t}$ denotes the energy transmission distance between nano-sensor $n$ and the PS during the WET stage, and $\vec{d}_{n}^{\sharp, t}$ indicates the data communications distance between the sink node and nano-sensor $n$ for the WIT stage in time slot $t$, as shown in Fig. 3. Note that a nano-sensor travels via Brownian motion with a drift. In this paper, we employ Langevin equation ${ }^{3}$ to describe the mobility of a nano-sensor. Motivated by [22], the dynamic energy transmission distance of nano-sensor $n$ is

$$
\frac{d \vec{d}_{n}^{\mathbb{E}, t}}{d t}=\frac{\beta_{n, \mathbb{E}}^{L}}{\alpha_{n, \mathbb{E}}^{L}} \frac{d \vec{W}_{n}(t)}{d t}
$$

\footnotetext{
${ }^{1}$ In this paper, the nano-sensor will send the power request when its available energy resource is below a preset threshold.

${ }^{2}$ The PS transmits the wireless power to nano-sensors in the WET stage via RF signals, while the nano-sensors forward the data packets in the WIT stage via terahertz bands.

${ }^{3}$ The Langevin equation was originally to describe the Brownian motion of a particle.
} 


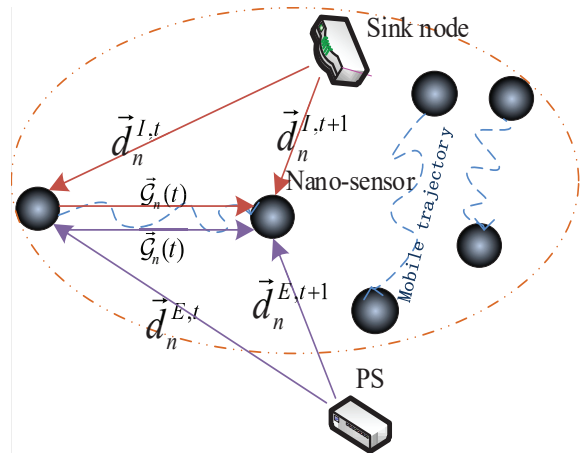

Fig. 3: Motion of mobile nano-sensors.

where $\beta_{n, \mathbb{E}}^{L}$ and $\alpha_{n, \mathbb{E}}^{L}$ are the positive constants determining the noise, the nano-sensor's resistance to motion, respectively. $\vec{W}_{n}(t)$ is the vector Wiener process. Then, we can obtain the dynamic energy transmission distance between nano-sensor $n$ and the PS for discrete time slot $t$, which is

$$
\vec{d}_{n}^{\mathbb{E}, t+1}=\vec{d}_{n}^{\mathbb{E}, t}+\frac{\beta_{n, \mathbb{E}}^{L}}{\alpha_{n, \mathbb{E}}^{L}} \overrightarrow{\mathcal{G}}(\tau),
$$

where $\overrightarrow{\mathcal{G}}(\tau)=\vec{W}(t+1)-\vec{W}(t)$ is a random Gaussian process and $\tau$ denotes the duration of one time slot. Similarly, the dynamic data communications distance between nano-sensor $n$ and the sink node for the discrete time slot $t$ can be described by

$$
\vec{d}_{n}^{\sharp, t+1}=\vec{d}_{n}^{\rrbracket, t}+\frac{\beta_{n, \mathbb{I}}^{L}}{\alpha_{n, \mathbb{I}}^{L}} \overrightarrow{\mathcal{G}}(\tau),
$$

where $\alpha_{n, \mathbb{I}}^{L}$ and $\beta_{n, \mathbb{I}}^{L}$ are positive constants.

\section{Dynamic Channel Model of the Terahertz Band for Data Transmission}

Data transmission in the terahertz band communications during the WIT stage for each time slot may incur significant path loss. $x_{n, m}^{t}$ is a binary variable indicator function. If $x_{n, m}^{t}=1$ it represents that nano-sensor $n$ transmitting its radio signal over channel $f_{m}$ in time slot $t$; otherwise, channel $f_{m}$ is idle over time slot $t$. Path loss of the terahertz band is jointly affected by the absorption loss $P L_{a b s}^{t}\left(x_{n, m}^{t}, \vec{d}_{n}^{\sharp, t}\right)$, and spread path loss $P L_{s p r}^{t}\left(x_{n, m}^{t}, \vec{d}_{n}^{\sharp, t}\right)$. Motivated from [23], the expression of the path loss in decibels $P L^{t}\left(x_{n, m}^{t}, \vec{d}_{n}^{\mathbb{1}, t}\right)$ of nano-sensor $n$ over frequency band $f_{m}$, can be expressed as

$$
\begin{aligned}
& P L^{t}\left(x_{n, m}^{t}, \vec{d}_{n}^{\mathbb{1}, t}\right)[d B] \\
& =P L_{s p r}^{t}\left(x_{n, m}^{t}, \vec{d}_{n}^{\sharp, t}\right)[d B]+P L_{a b s}^{t}\left(x_{n, m}^{t}, \vec{d}_{n}^{\sharp, t}\right)[d B] \\
& =20 \log \left(\frac{4 \pi \vec{d}_{n}^{\mathbb{1}, t} x_{n, m}^{t} f_{m}}{c}\right)[d B]+10 K_{t, x_{n, m}^{t}, \vec{d}_{n}^{\mathbb{1}, t}}^{n} \log (e)[d B],
\end{aligned}
$$

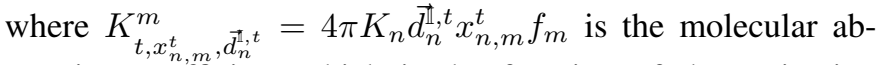
sorption coefficient which is the function of the extinction $K_{m} . x_{n, m}^{t} f_{m}$ is the operating channel of nano-sensor $n$ and $c$ is the speed of light. From Eq. (4), it is clear that the path loss $P L^{t}\left(x_{n, m}^{t}, \vec{d}_{n}^{\mathbb{1}, t}\right)$ is the function of the terahertz frequency, and that the frequency selective feature is dominant for transmissions involving longer distances $\vec{d}_{n}^{\sharp, t}$. The noise for nano-sensor $n$ in the terahertz band is primarily due to the molecular absorption noise, which is given by

$$
N_{a b s}^{n}\left(t, x_{n, m}^{t}, \vec{d}_{n}^{\mathbb{1}, t}\right)=k_{B} T_{0}\left(1-e^{-K_{t, x_{n, m}^{t}, d_{n}^{\mathbb{I}}, t}^{n}}\right),
$$

where $T_{0}$ is the reference temperature, $k_{B}$ is the Boltzmann constant. If $S_{n, m}^{t}$ is the power spectral density of the transmitted radio signal in time slot $t$ over channel $f_{m}$. Then, the SNR of nano-sensor $n$ over $f_{m}$ and distance $\vec{d}_{n}^{\mathbb{1}, t}$ in time slot $t$ is given as below:

$$
\begin{aligned}
& S N R_{n}^{m}\left(t, x_{n, m}^{t}, \vec{d}_{n}^{\sharp, t}\right)=\frac{S_{n, m}^{t}}{P L^{t}\left(x_{n, m}^{t}, \vec{d}_{n}^{\mathbb{1}, t}\right) N_{a b s}^{n}\left(t, x_{n, m}^{t}, \vec{d}_{n}^{\mathbb{1}, t}\right)} \\
& =\frac{S_{n, m}^{t}}{\Psi\left(t, \vec{d}_{n}^{\sharp, t}, x_{n, m}^{t}\right)},
\end{aligned}
$$

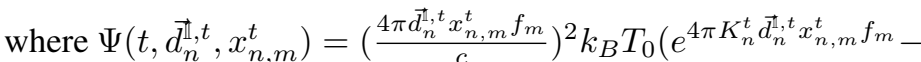
$1)$. As each nano-sensor operates over an orthogonal channel. Then, we have the following constraint,

$$
\sum_{n} x_{n, m}^{t} \leq 1, x_{n, m}^{t} \in\{0,1\}, \forall m \in \mathcal{M} .
$$

Where $\sum_{n}=\sum_{1}^{N}$. The transmission rate of nano-sensor $n$ towards the sink node over time slot $t$ can be represented with the help of Shannon capacity formula as follows:

$r_{n}^{m}(t)=\triangle f \log \left(1+S N R_{n}^{m}\left(t, x_{n, m}^{t}, \vec{d}_{n}^{\mathbb{1}, t}\right)\right), \forall t>0, n \in \mathcal{N}$.

Where $\triangle f$ is the width of each terahertz channel band.

\section{PROBLEM REFORMULATION}

\section{A. Problem Formulation}

In this section, we discuss the joint consideration the longterm channel capacity and energy consumption to ensure the effective data transmission in nano-empowered vehicular networks.

1) Energy Consumption and Supply for Nano-sensors: In this paper, we employ WPT technology in nano-communications networks. A nano-sensor's energy resource will change with the energy transfer process during the WET stage and with the data communications process during the WIT stage. Note that, for the WET stage, when the PS receives energy requests, it will send power resources to nano-sensors via RF signals. Based on [24], we know that the energy transfer model from the PS to the nano-sensor encompasses both the distancedependent path loss and small-scale fading. Then, the channel power gain for energy transmission to nano-sensor $n, h_{n}^{t}$, is calculated using the following formula [25],

$$
h_{n}^{t}=10^{-\frac{P L^{t}\left(\vec{d}_{n}^{\mathbb{1}, t}\right)[d B]+\psi_{n}^{t}[d B]}{10}} \vec{d}_{n}^{\mathbb{1}, t} \beta_{n}^{t},
$$

where $P L^{t}\left(\vec{d}_{n}^{\mathbb{1}, t}\right)$ is the path loss in decibels for distance $\vec{d}_{n}^{\mathbb{1}, t}$, $\psi_{n}^{t}$ is the loss margin between the PS and nano-sensor $n$ in decibels, and $\beta_{n}^{t}$ is the small-scale fading power gain between the PS and nano-sensor $n$. During the WET stage in time slot $t$, the PS is assumed to transmit $e_{n}^{t}$ wireless power, where 
$0 \leq e_{n}^{t} \leq o_{n}$, to nano-sensor $n$. Then, the amount of power received for nano-sensor $n$ from the PS in time slot $t$ is

$$
C_{c a p^{n}}^{t}=\varsigma_{n}^{t}\left(T_{0}\right) e_{n}^{t} h_{n}^{t} \theta \tau,
$$

where $\varsigma_{n}^{t}\left(T_{0}\right)$ is the power transfer efficiency at nano-sensor $n$. Then, the energy consumption of nano-sensor $n$ in the WIT stage for time slot $t$ is

$$
E_{n}^{W E T}(t)=P C_{n} \theta \tau-C_{c a p^{n}}^{t},
$$

where $P C_{n}$ is the constant circuit power consumption of nanosensor $n$.

During the WIT stage, each nano-sensor transmits the data packets in its communication block to the sink. Motivated by [26], the energy required to handle the transmission for the WIT stage is given by $E_{T r}^{n}(t)=\sum_{m} x_{n, m}^{t} S_{n, m}^{t}(1-\theta) \tau$. The energy consumption for nano-deceive $n$ receiving packets during $(1-\theta) \tau$ is $E_{R e}^{n}(t)=p_{r}^{t}(1-\theta) \tau$, where the nanosensor's receive power $p_{r}^{t}$ is assumed to be constant in this paper. Then, the energy consumption of nano-sensor $n$ during the WIT stage in time slot $t$ is

$$
\begin{aligned}
& E_{n}^{W I T}(t)=E_{R e}^{n}(t)+E_{T r}^{n}(t)+P C_{n}(1-\theta) \tau \\
= & \left(\sum_{m} x_{n, m}^{t} S_{n, m}^{t}+P C_{n}+p_{r}^{t}\right)(1-\theta) \tau .
\end{aligned}
$$

where $E_{n}^{t}$ is a positive value. Moreover, to ensure the energy balance among the nano-sensors, we shall consider the different energy levels of the nano-sensor. Noting that, for their energy consumptions to execute data transmission, the nanosensor with less energy resource will pay relative more price than others. We define the nano-sensor's energy consumption considering the current energy state as its relative energy cost. Then, the related energy costs for nano-sensors in overall time slot $t$ is

$$
\begin{aligned}
& P_{t}^{\text {total }}\left(\mathbf{x}^{t}, \mathbf{S}^{t}\right)=\sum_{n} \frac{E_{n}^{W E T}(t)+E_{n}^{W I T}(t)}{E_{n}^{t}} \\
= & \sum_{n} \frac{P C_{n}+\left(\sum_{m} x_{n, m}^{t} S_{n, m}^{t}+p_{r}^{t}\right)(1-\theta) \tau-C_{c a p^{n}}^{t}}{E_{n}^{t}} .
\end{aligned}
$$

where $\mathbf{x}^{t}=\left\{x_{n, m}^{t}\right\}_{n \in \mathcal{N}, m \in \mathcal{M}}, \mathbf{S}^{t}=\left\{S_{n, m}^{t}\right\}_{n \in \mathcal{N}, m \in \mathcal{M}}$. The notation $E_{n}^{t}$ is nano-sensor $n$ 's energy state in time slot $t$, which is updated as

$$
E_{n}^{t+1}=\max \left\{0, E_{n}^{t}-E_{n}^{W E T}(t)-E_{n}^{W I T}(t)\right\},
$$

where $E_{n}^{t}$ is a positive value.

2) Channel Capacity: In this section, with the help of Shannon capacity formula, we can present overall terahertz channel capacity as a summation of each sub-channel over time slot $t$, given as follows:

$$
\begin{aligned}
C_{T H z}\left(\mathbf{x}^{t}, \mathbf{S}^{t}\right) & =\sum_{n} \sum_{m} \triangle f \log _{2}\left(1+S N R_{n}^{m}\left(t, x_{n, m}^{t}, \vec{d}_{n}^{\sharp, t}\right)\right) \\
& =\sum_{n} \sum_{m} \triangle f \log _{2}\left(1+\frac{S_{n, m}^{t}}{\Psi\left(t, \vec{d}_{n}^{\mathbb{1}, t}, x_{n, m}^{t}\right)}\right),
\end{aligned}
$$

where $\log _{2}$ is the binary logarithm.

Motivated by [27] and [28], the energy efficiency (EE) $\rho_{E E}^{\text {total }}$ for the nano-communication networks is defined as the ratio of the long-term sum channel capacity to the corresponding long-term total related energy costs, given as,

$$
\rho_{E E}^{\text {Total }}=\frac{\sum_{t=1}^{\infty} C_{T H z}\left(\mathbf{x}^{t}, \mathbf{S}^{t}\right)}{\sum_{t=1}^{\infty} P_{t}^{\text {total }}\left(\mathbf{x}^{t}, \mathbf{S}^{t}\right)},
$$

Compared with the traditional EE, the EE defined in this paper benefits from the energy balance among the nano-sensors. According to EE definition discuss above, we conclude the larger $\rho_{E E}^{T o t a l}$ is desirable. As, our objectives is to maximize the long-term $\mathrm{EE} \rho_{E E}^{T \text { otal }}$ by jointly considering the power constraints and channel property of $\mathrm{THz}$ band. Thus, the longterm EE optimization problem can be formulated as,

$$
\begin{aligned}
& \text { P1: } \max _{\mathbf{x}^{t}, \mathbf{S}^{t}} \rho_{E E}^{\text {Toatl }}=\frac{\sum_{t=1}^{\infty} C_{T H z}\left(\mathbf{x}^{t}, \mathbf{S}^{t}\right)}{\sum_{t=1}^{\infty} P_{t}^{\text {total }}\left(\mathbf{x}^{t}, \mathbf{S}^{t}\right)} \\
& \text { s.t. } \sum_{n} x_{n, m}^{t} \leq 1, x_{n, m}^{t} \in\{0,1\}, \forall m \in \mathcal{M} \text {, } \\
& \sum_{n} \sum_{m} x_{n, m}^{t} S_{n, m}^{t} \leq P_{\max }, \forall t \geq 0 .
\end{aligned}
$$

In the problem P1, the Constraint (C1) ensure the orthogonality of terahertz channels allocated to nano-sensors; (C2) denotes the total energy constraint. Due to the fractional forms of the EE function, the Problem P1 is a non-convex optimization problem, that cannot be solved by the conventional convex optimization techniques. Moreover, the binary variables $\mathbf{x}^{t}$ makes textbfP1 a mixed-integer non-linear programming (MINLP) that is hard to solve [29].

\section{B. Resource Allocation Problem in Time Slot $t$}

P1 can be classified as a non-linear fractional program with the definition of EE. In this subsection, we will convert problem P1 into a series of solvable resource allocation problems for $\mathrm{EE}$ over the time slots, and propose the energy-efficient resource allocation problem in a specific time slot.

Noting that, the optimal $E E$ is defined as $\rho_{E E}^{\text {Total }}{ }^{*}=$ $\frac{\sum_{t=1}^{\infty} C_{T H z}\left(\mathbf{x}^{t^{*}}, \mathbf{S}^{t^{*}}\right)}{\sum_{t=1}^{\infty} P_{t}^{\text {total }}\left(\mathbf{x}^{t^{*}}, \mathbf{S}^{t^{*}}\right)}$, where $\mathbf{x}^{t^{*}}$ and $\mathbf{S}^{t^{*}}$ are the optimal solutions of problem P1 in time slot $t$. Then, based on the equivalent transformation method [30], the resource allocation decisions can achieve the optimal EE $\rho_{E E}^{T o t a l}{ }^{*}$ if and only if

$\sum_{t=1}^{\infty} C_{T H z}\left(\mathbf{x}^{t^{*}}, \mathbf{S}^{t^{*}}\right)-\rho_{E E}^{\text {Total }} \sum_{t=1}^{\infty} P_{t}^{\text {total }}\left(\mathbf{x}^{t^{*}}, \mathbf{S}^{t^{*}}\right)=0$.

From above, we know that if the optimal value $\rho_{E E}^{T o t a l^{*}}$ is given, problem P1 is equivalent to the following optimization problem,

$$
\begin{aligned}
\text { P2: } \max & \sum_{t=1}^{\infty} C_{T H z}\left(\mathbf{x}^{t}, \mathbf{S}^{t}\right)-\rho_{E E}^{\text {Total }}{ }^{*} \sum_{t=1}^{\infty} P_{t}^{\text {total }}\left(\mathbf{x}^{t}, \mathbf{S}^{t}\right) \\
\text { s.t. } & (\mathrm{C} 1) \text { and }(\mathrm{C} 2) .
\end{aligned}
$$

Problem P2 is a long-term optimization problem, which is also difficult to solve. By defining $\rho_{E E}^{t}$ as the EE over the past interval $[0, t]$, we obtain the following theorem for the convergence performance of $\rho_{E E}^{t}$. 
Theorem 1. Updating $\rho_{E E}^{t}$ as

$$
\rho_{E E}^{t}=\frac{\sum_{t=1}^{t} C_{T H z}\left(\mathbf{x}^{t}, \mathbf{S}^{t}\right)}{\sum_{t=1}^{t} P_{t}^{\text {total }}\left(\mathbf{x}^{t}, \mathbf{S}^{t}\right)}
$$

for each time slot, $\eta_{E E}^{t}$ will gradually reach to $\rho_{E E}^{\text {Total }}{ }^{*}$. The resource allocation decisions $\left(\mathrm{x}^{t}\right.$ and $\left.\mathbf{S}^{t}\right)$ in the equation above obtained via

$$
\max _{\mathbf{x}^{t}, \mathbf{S}^{t}} C_{T H z}\left(\mathbf{x}^{t}, \mathbf{S}^{t}\right)-\rho_{E E}^{t} P_{t}^{\text {total }}\left(\mathbf{x}^{t}, \mathbf{S}^{t}\right)
$$

Proof. Similar proof can be found in [30].

After the transformation, the difficulty for solving problem P1 turns into solving Eq. (18) under a given $\rho_{E E}^{t}$. Then, the energy-efficient resource allocation problem for nano-sensors in time slot $t$ can be written as

$$
\text { P3: } \begin{aligned}
\max _{\mathbf{x}^{t}, \mathbf{S}^{t}} & C_{T H z}\left(\mathbf{x}^{t}, \mathbf{S}^{t}\right)-\rho_{E E}^{t} P_{t}^{\text {total }}\left(\mathbf{x}^{t}, \mathbf{S}^{t}\right) \\
= & \sum_{n}\left\{\begin{array}{c}
\sum_{m} \triangle f \log _{2}\left(1+\frac{S_{n, m}^{t}}{\Psi\left(t, \vec{d}_{n}^{n, t}, x_{n, m}^{t}\right)}\right) \\
-\frac{\rho_{E E}^{t}(1-\theta)}{E_{n}^{t}} \sum_{m} x_{n, m}^{t} S_{n, m}^{t}-\frac{\rho_{E E}^{t}}{E_{n}^{t}} \Phi_{n}(t)
\end{array}\right\}
\end{aligned}
$$$$
\text { s.t. (C1) and (C2), }
$$

where $\Phi(t)=P C_{n}+p_{r}^{t}(1-\theta) \tau-C_{c a p^{n}}^{t}$. Problem $\mathbf{P 3}$ is an MINLP problem, and is dependent on variables $\mathbf{x}^{t}$ and $\mathbf{S}^{t}$.

A simple way to get the MINLP problem solution is to use exhaustive search [29]. However, this is computationally expensive and intractable. Recently, two approximate methods are proposed for the sub-optimal solution of the MINLP: the branch-and-bound method [18], and the continuous convex approximation [17]. However, the sufficient condition of using the approximate methods above is that the MINLP problem can be transformed into a convex optimization problem. Thus, due to the complex properties of of terahertz channel it is hard to convert P3 into a convex optimization problem. Therefore, sub-optimal solution of the problem is derived by using the heuristic approach with acceptable time complexity. In the following section, we will employ an intelligence method to solve problem P3, and propose two dynamic EE optimization algorithms to obtain the sub-optimal solution for problem P3.

\section{DYNAMIC EE OPTIMIZATION ALGORITHMS}

Note that PSO method [31] is a kind of swarm intelligence technology, which is suitable for solving the complicated optimization problems. In this section, we first employ quantumbehaved particle swarm optimization (QPSO) method, which is a new version of PSO, to obtain the sub-optimal solution to problem P3. Then, a dynamic PSO-based EE optimization algorithm (DPEEO) for problem P1 is proposed in harmony with the time-varying network state. Based on the special structures of the objective function and the constraints in problem P3, an improved DPEEO algorithm is presented, which performs better in handing the constraints and reducing the length of the convergence time.

\section{A. Dynamic PSO-Based EE Optimization Algorithm}

From the perspective of quantum mechanism, QPSO algorithm is proposed whose global search ability is better than the standard PSO. Number of quantum particles in a swarm for QPSO method are represented by $J$. The solution can be obtained from the position of each individual quantum particle $O_{j}, j \in J$, where $O_{j}, j \in J$ gradually move towards the optimal position by increasing the number of iterations. Thus, the fitness function is opted to evaluate the quality of a quantum particle's position. The detailed process of the QPSO method for problem $\mathbf{P 3}$ is as follows.

1) Positions for the quantum particle: The exact position and velocity values cannot be determined simultaneously due to the uncertainty principle [32]. As a consequence, only the position vector is defined in the QPSO method. Specially, in the $i$-th iteration, the position of quantum particle $O_{j}$ in the swarm is denoted by

$$
\mathbf{Z}_{t}^{j, i}=\left[\left\{x_{n, m}^{t}{ }^{j, i}\right\}_{n \in \mathcal{N}, m \in \mathcal{M}},\left\{S_{n, m}^{t}{ }^{j, i}\right\}_{n \in \mathcal{N}, m \in \mathcal{M}}\right] .
$$

The previous best position for particle $O_{j}$ and all particles respectively, are

$$
p \mathbf{Z}_{t}^{j, i}=\left[\left\{p x_{n, m}^{t}{ }^{j, i}\right\}_{n \in \mathcal{N}, m \in \mathcal{M}},\left\{p S_{n, m}^{t}{ }^{j, i}\right\}_{n \in \mathcal{N}, m \in \mathcal{M}}\right],
$$

and

$$
g \mathbf{Z}_{t}^{i}=\left[\left\{g x_{n, m}^{t}{ }^{i}\right\}_{n \in \mathcal{N}, m \in \mathcal{M}},\left\{g S_{n, m}^{t}{ }^{i}\right\}_{n \in \mathcal{N}, m \in \mathcal{M}}\right] .
$$

The position for particle $O_{j}$ is updated based on the following iterative equation

$$
\mathbf{Z}_{t}^{j, i+1}= \begin{cases}\mathbf{B}_{t}^{j, i}+\beta\left|m \mathbf{Z}_{t}^{i}-\mathbf{Z}_{t}^{j, i}\right| \ln \left(\frac{1}{\xi}\right) & \text { if } \varepsilon \geq 0.5 \\ \mathbf{B}_{t}^{j, i}-\beta\left|m \mathbf{Z}_{t}^{i}-\mathbf{Z}_{t}^{j, i}\right| \ln \left(\frac{1}{\xi}\right) & \text { otherwise }\end{cases}
$$

where $\xi$ and $\varepsilon$ are the random numbers between 0 and $1 . \beta$ is the contraction-expansion coefficient, which can be calculated by $\beta=1-\frac{i}{2 \text { inter }^{\max }}$ where inter $^{\max }$ is the maximum number of iterations. In addition, the local attractor $\mathbf{B}_{t}^{j, i}$ is acquired by

$$
\mathbf{B}_{t}^{j, i}=\eta p \mathbf{Z}_{t}^{j, i}+(1-\eta) g \mathbf{Z}_{t}
$$

and the mean position for all particle is

$$
m \mathbf{Z}_{t}^{i}=\frac{1}{J} \sum_{j} p \mathbf{Z}_{t}^{j, i}
$$

where $\eta$ is a random weight between 0 and 1 .

2) Fitness for the quantum particle: In order to apply the QPSO method to solve problem P3, the original constrained optimization problem needs to be transformed into an unconstrained form, which can be done with the penalty function method. Thus, motivated by [33], a fitness function in the $i$ th iteration that consists of one objective function and one penalty function is constructed in Eq. (23) at the top of the next page, where $\gamma$ denotes the penalty factor, and $\mathcal{P}_{f}\left(x_{n, m}^{t}, S_{n, m}^{t}\right)$ indicates the penalty function that includes two items, i.e.,

$\mathcal{P}_{f}\left(x_{n, m}^{t}, S_{n, m}^{t}\right)=\left(\sum_{n} \sum_{m} x_{n, m}^{t} S_{n, m}^{t}-P_{\max }\right)+\left(\sum_{n} x_{n, m}^{t}-1\right)$.

In terms of the time-varying network state, we propose a DPEEO algorithm, whose details are shown in Algorithm 1. 
$\operatorname{Fitness}\left(\mathbf{Z}_{n}^{j, i}\right)=\sum_{n} \sum_{m}\left[\triangle f \log _{2}\left(1+\frac{S_{n, m}^{t}{ }^{j, i}}{\Psi\left(t, \vec{d}_{n}^{\mathbb{1}, t}, x_{n, m}^{t}{ }^{j, i}\right)}\right)-\frac{\rho_{E E}^{t}(1-\theta)}{E_{n}^{t}} x_{n, m}^{t}{ }^{j, i} S_{n, m}^{t}{ }^{j, i}-\gamma \mathcal{P}_{f}\left(x_{n, m}^{t}{ }^{j, i}, S_{n, m}^{t}{ }^{j, i}\right)\right]-\sum_{n} \frac{\rho_{E E}^{t}}{E_{n}^{t}} \Phi_{n}(t)$,

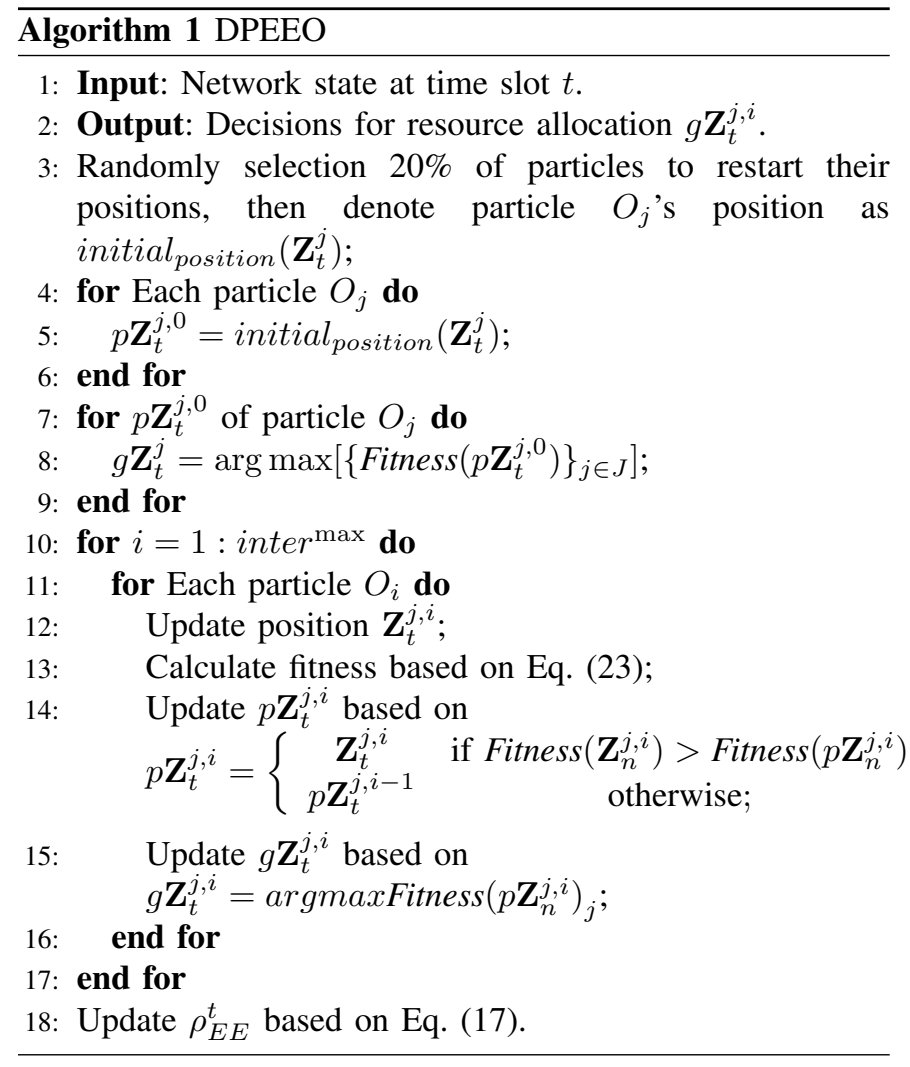

Remark 1. By virtue of the coherence of the network state between two adjacent timeslots, 20\% of the particles are randomly selected to restart their positions, and the initial position for each particle in the swarm is generated (Step 3). If the number of iterations reaches inter ${ }^{\max }$, the solution is obtained, and the run is terminated.

\section{B. Improved Dynamic PSO-Based EE Optimization Algorithm}

From Algorithm 1, the sub-solution of problem $\mathbf{P 1}$ is achieved. However, some things remain to be enhanced: a) In Algorithm 1, we employ a penalty function to handle the constraint in problem P3. Then, the algorithm's performance is significantly affected by the value of penalty parameter $\gamma$. Particles can enter into the feasible region quickly if $\gamma$ value is large enough. However, this may leads to miss some valuable information in the infeasible region, which could result in the algorithm being trapped in local minima. While, a toosmall $\gamma$ may induce an unacceptable infeasible solution. b) In Algorithm 1, the dimensionality of the variable for a particle is $(2 N) \times M$, which means each particle has to potentially search a larger area in the available space. Then, the swarm may lose its diversity in some dimensions, and Algorithm 1 may suffer from the curse of dimensionality, which greatly deteriorates its performance.

We can obtain the relationship between the power control
$S_{n, m}^{t}$ and frequency selection $x_{n, m}^{t}$, based on the property of the objective function in $\mathbf{P 3}$, and then can develop a new optimization problem for variables $\mathrm{x}^{t}$. By jointly solving the new problem for variables $\mathbf{x}^{t}$ and exploiting the relationship between variables, we propose an improved DPEEO algorithm for problem P1. The proposed algorithm can handle the constraint of the problem quite well, and greatly decreases the length of time required to convergence to the optimal solution. The details are illustrated as follows.

1) Relationship between frequency selection and power control: In P3, we first relaxes $x_{n, m}^{t}$ over a continuous interval $[0,1]$, which is represented by $\breve{x}_{n, m}^{t}$, and then introduce a new variable, $\breve{S}_{n, m}^{t}=\breve{x}_{n, m}^{t} S_{n, m}^{t}$ based on the primal-dual decomposition method [34]. Then, we can rewrite $\mathbf{P 3}$ as

$$
\begin{aligned}
\max _{\breve{\mathbf{S}}^{t}} & \sum_{n} \sum_{m} \triangle f \log _{2}\left(1+\frac{\breve{S}_{n, m}^{t}}{\breve{x}_{n, m}^{t} \Psi\left(t, \vec{d}_{n}^{\mathbb{1}, t}, \breve{x}_{n, m}^{t}\right)}\right) \\
& -\sum_{n} \frac{\rho_{E E}^{t}(1-\theta)}{E_{n}^{t}} \sum_{m} \breve{S}_{n, m}^{t}-\sum_{n} \frac{\rho_{E E}^{t}}{E_{n}^{t}} \Phi_{n}(t) \\
\text { s.t. } & \sum_{m} \breve{x}_{n, m}^{t} \leq 1,0 \leq \breve{x}_{n, m}^{t} \leq 1, \quad(\mathrm{C} 3) \\
& \sum_{n} \sum_{m} \breve{S}_{n, m}^{t} \leq P_{\max }, \forall t \geq 0 . \quad(\mathrm{C} 4)
\end{aligned}
$$

The Eq. (25) is the convex optimization problem over variable $\breve{S}_{n, m}^{t}$, in which constraint (C3) is an affine function and feasible set of constraints is a convex set. Thus, we can derive the relationship between the variable $\breve{x}_{n, m}^{t}$ and $\breve{S}_{n, m}^{t}$ and by solving Eq. (25) according to gradient descent (GD) method introduced in [35]. Moreover, by introducing the Lagrange multiplier $u$, we can relax the power constraint in Eq. (25) and can get the Lagrange function from Eq. (26).

When using the Karush-Kuhn-Tucker (KKT) conditions on Eq. (26), the relationship between $\breve{S}_{n, m}^{t}$ and $\breve{x}_{n, m}^{t}$ can be written as

$$
\breve{S}_{n, m}^{t}=\left(\frac{\triangle f}{u+\frac{\rho_{E E}^{t}(1-\theta)}{E_{n}^{t}}}-\Psi\left(t, \vec{d}_{n}^{\sharp, t}, \breve{x}_{n, m}^{t}\right)\right) \breve{x}_{n, m}^{t} .
$$

2) Improved dynamic PSO-based EE optimization algorithm: We can obtain Eq. (28) for binary variable $x_{n, k}^{m}$, by substituting Eq. (27) into Eq. (25) which is a typical nonlinear 0-1 integer programming problem.

The overlapping of frequencies $\sum_{n} x_{n, m}^{t} \leq 1, \forall m \in \mathcal{M}$ are avoided in Eq. (28) which means that one frequency band or terahertz channel can only be assigned to one nano-sensor. Thus, we can rewrite frequency allocation $\mathbf{x}_{t}$ as a new variable $\mathbf{a}_{t}=\left[\left\{a_{m}^{t}\right\}_{m \in M}\right]$. Among it, the element $a_{m}^{t}$ taking value from the set $[0, N]$, denotes which nano-sensor the frequency $f_{m}$ is allocated to. Especially, when $a_{m}^{t}=0$, it means the $f_{m}$ is not occupied by any of nano-sensor. The notation $\mathbf{a}_{t}$ naturally prevents the overlapping of frequencies. In this way, 


$$
L\left(\mathbf{S}, u_{n}\right)=\sum_{n} \sum_{m} \triangle f \log _{2}\left(1+\frac{\breve{S}_{n, m}^{t}}{\breve{x}_{n, m}^{t} \Psi\left(t, \vec{d}_{n}^{\mathbb{1}, t}, x_{n, m}^{t}\right)}\right)-\frac{\rho_{E E}^{t}(1-\theta)}{E_{n}^{t}} \breve{S}_{n, m}^{t}-\sum_{n} \frac{\rho_{E E}^{t}}{E_{n}^{t}} \Phi_{n}(t)+u\left(\sum_{n} \sum_{m} \breve{S}_{n, m}^{t}-P_{\max }\right) .
$$

$$
\begin{aligned}
\max _{\mathbf{x}} & \sum_{m} \sum_{n} \triangle f \log _{2}\left(\frac{\triangle f}{\left(u+\frac{\rho_{E E}^{t}(1-\theta)}{E_{n}^{t}} \Psi\left(t, \vec{d}_{n}^{\mathbb{1}, t}, x_{n, m}^{t}\right)\right.}\right)-\sum_{n} \frac{\rho_{E E}^{t}(1-\theta)}{E_{n}^{t}} \sum_{m}\left(\frac{\triangle f}{u+\frac{\rho_{E E}^{t}(1-\theta)}{E_{n}^{t}}}-\Psi\left(t, \vec{d}_{n}^{\sharp, t}, x_{n, m}^{t}\right)\right) x_{n, m}^{t}-\sum_{n} \frac{\rho_{E E}^{t}}{E_{n}^{t}} \Phi_{n}(t) \\
\text { s.t. } & \sum_{n} \sum_{m} x_{n, m}^{t} \leq 1, x_{n, m}^{t} \in\{0,1\} .
\end{aligned}
$$

we can convent Eq. (28) into an unconstrained optimization problem, and employ an improved PSO method to find the sub-optimal frequency allocation by expanding the basic PSO in a binary space. Similarly, $J$ is used to denote the number of quantum particles in a swarm for the improved PSO method. In the $i$-th iteration, the position and velocity of the $j$-th particle in the swarm can be denoted by

$$
\mathbf{a}_{t}^{j, i}=\left[\left\{a_{m}^{t}{ }^{j, i}\right\}_{m \in \mathcal{M}}\right] \text {, and } \tilde{\mathbf{V}}_{t}^{j, i}=\left[\left\{\tilde{V}_{m}^{j, i}\right\}_{m \in \mathcal{M}}\right] .
$$

The previous best positions for the $j$-th particle and all particles in the $i$-th iteration respectively are

$$
p \mathbf{a}_{t}^{j, i}=\left[\left\{p a_{m}^{t}{ }^{j, i}\right\}_{m \in \mathcal{M}}\right] \text {, and } g \mathbf{a}_{t}^{i}=\left[\left\{g a_{n, m}^{t}{ }^{i}\right\}_{m \in \mathcal{M}}\right] .
$$

The new velocity of each particle can be found as follows,

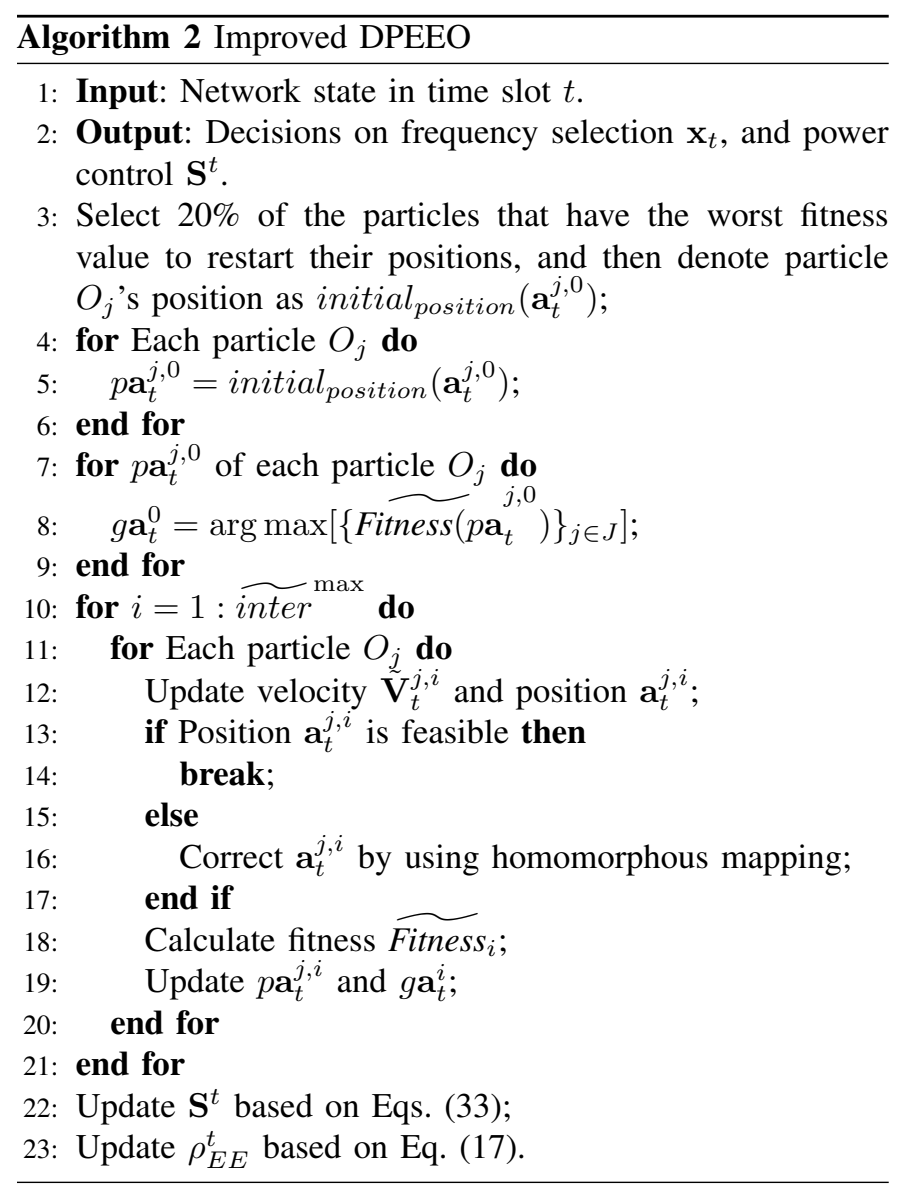

$\tilde{\mathbf{V}}_{t}^{j, i+1}=\varpi_{j, i} \tilde{\mathbf{V}}_{t}^{j, i+1}+c_{1} r_{1}\left(p \mathbf{a}_{t}^{j, i}-\mathbf{a}_{t}^{j, i}\right)+c_{2} r_{2}\left(g \mathbf{a}_{t}^{i}-\mathbf{a}_{t}^{j, i}\right)$ where $r_{1}$ and $r_{2}$ are two independent random numbers uniformly distributed over $[0,1]$ and $c_{1}$ and $c_{2}$ denotes the acceleration coefficients constants. inertia factor $\varpi_{j, i}$ is as follows:

$$
\varpi_{j, i}=\varpi_{\max }-\frac{i\left(\varpi_{\max }-\varpi_{\min }\right)}{\widetilde{\text { inter }}}
$$

where $\widetilde{\text { inter }}^{\max }$ denotes the maximum number of iterations and $\varpi_{\min }$ and $\varpi_{\max }$ represent the minimum and maximum inertia weights, respectively. Then, the position $\mathbf{x}_{t}^{j, i}$ for particle $O_{j}$ in the $i$-th iteration is updated as

$a_{n}^{t}{ }^{j, i+1}=\left\{\begin{array}{cc}{\left[\mathbf{x}_{t}^{j, i}+\tilde{\mathbf{V}}_{m}^{j, i+1}\right]^{-}} & \text {if } \bmod \left(\mathbf{x}_{t}^{j, i}+\tilde{\mathbf{V}}_{n}^{j, i+1}\right)>\operatorname{rand}_{m}^{j, i}, \\ {\left[\mathbf{x}_{t}^{j, i}+\tilde{\mathbf{V}}_{m}^{j, i+1}\right]^{-}+1} & \text { otherwise },\end{array}\right.$

The fitness function is constructed in Eq. (32). Whereas, $[\cdot]^{-}$ is the maximal integer which is less than the value in $[\cdot]$. Thus, optimal frequency allocation $\mathrm{x}_{t}$ is achieved using the optimal variable $g \mathbf{a}_{t}$. Thus, we can further, obtain optimal power control $S_{n, m}^{t}$ for the $i$-th iteration with the help of relationship established between variables in Eq. (27) which is as follows:

$$
S_{n, m}^{t}=\left\{\begin{array}{cc}
0 & \text { if } x_{n, m}^{t}=0, \\
\frac{\triangle f}{u+\frac{\rho_{E E}^{t}(1-\theta)}{E_{n}^{t}}}-\Psi\left(t, \vec{d}_{n}^{\|, t}, 1\right) & \text { otherwise. }
\end{array}\right.
$$

In terms of dynamic network state, an improved DPEEO algorithm is shown in Algorithm 2. 20\% of the particles that have the worst fitness valves are selected to restart their positions, which can make the algorithm achieve the optimal values more quickly. In Step 3, the initial position of each swarm particle over time slot $t$ is generated. From Steps 4-21, the relevant velocities and positions of the swarm particles are updated until it reaches to $\widetilde{\text { inter }}$ max , iterations. From Step 1317, we corrected the invalid positions, in case when the particle may be out of range with the help of homomorphous mapping [36]. This is more efficient method than the general schemes in which invalid positions are discarded simply. Furthermore, the power control variable is achieved based on Eq. (42) (Step 22), and the EE $\rho_{E E}^{t}$ is updated based on Eq. (17) (Step 23).

Remark 2. In this subsection, to tackle the original stochastic optimization problem in problem P3, we employ continuity relaxation and obtain Eq. (25). Considering the convex property of variables $\breve{S}_{n, m}^{t}$ in Eq. (25), the relationship between power control and frequency selection is achieved using the 


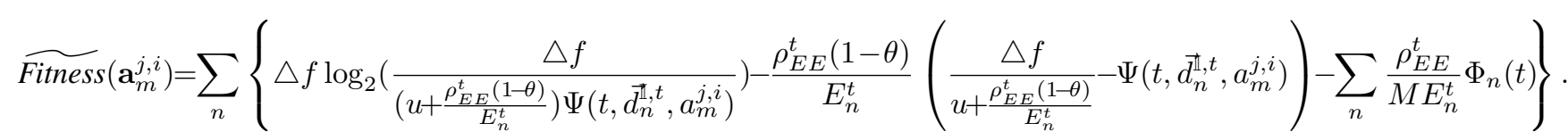

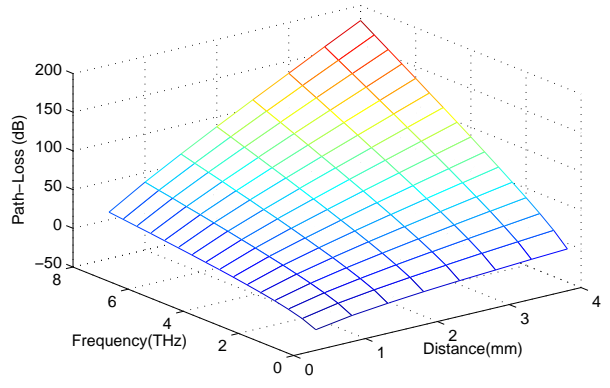

(a) Path loss

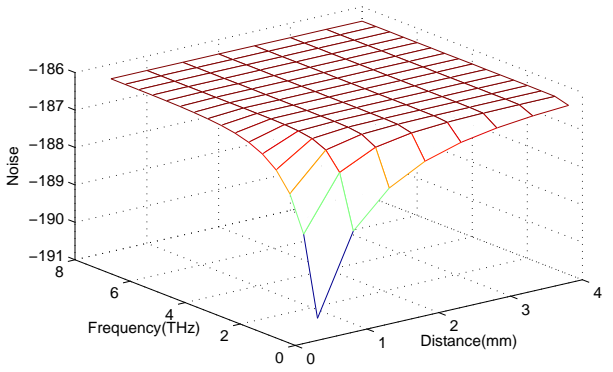

(b) Noise

Fig. 4: Channel property of terahertz band.

GD method. Submitting the obtained relationship into Eq. (25), a new problem is built in Eq. (28). Eq. (28) is a typical nonlinear 0-1 integer programming problem, which is difficult to solve. In terms of the property of constraint in Eq. (28), we translate Eq. (28) as an unconstrained optimal problem and employ an improved PSO method to solve. Then, Algorithm 2 is proposed to find the solution to problem P1. In this way, the dimensionality of the variables is reduced from $(2 N) \times M$ for mixed variables to $M$ for integer variables, which greatly decreases the computational complexity of the system. Compared with Algorithm 1, Algorithm 2 can handle the constraints quite well, decreases the research space, and greatly reduces the length of the convergence time.

\section{SIMULATION RESULTS}

In this section, we first provide the channel property of terahertz band. Then, the performance of the proposed algorithms are given.

1) Parameter setting: For the simulations, there are six nanosensors with eight discrete $\mathrm{THz}$ sub-bands, i.e, $N=6$ and $M=8$. The nano-sensors start to transmission with following energy resource: $E_{1}^{0}=60 p J, E_{2}^{0}=50 p J, E_{3}^{0}=40 p J$, $E_{4}^{0}=30 p J, E_{5}^{0}=20 p J$ and $E_{6}^{0}=10 p J$. The time is advanced in discrete slots of 5 seconds, i.e., $\tau=5 \mathrm{~s}$. In each time slot of a simulation, the nano-sensor undergoes random walks [37]. Moreover, by sampling Gaussian random variables
TABLE I: Algorithm performance

\begin{tabular}{|l|l|l|l|}
\hline Approach & $\begin{array}{l}\text { Network } \\
\text { size }\end{array}$ & $\mathbf{E E}$ & $\begin{array}{l}\text { Convergence } \\
\text { time (s) }\end{array}$ \\
\hline Normal PSO & & 6.4107 & 3.718 \\
DPEEO & $(\mathrm{N}=6, \mathrm{M}=8)$ & 6.4110 & 0.797 \\
IDPEEO & & $\mathbf{6 . 4 1 1 0}$ & $\mathbf{0 . 6 8 4}$ \\
\hline Normal PSO & & 6.6905 & 17.498 \\
DPEEO & $(\mathrm{N}=14, \mathrm{M}=16)$ & 6.6920 & 2.182 \\
IDPEEO & & $\mathbf{6 . 6 9 2 4}$ & $\mathbf{0 . 8 8 2}$ \\
\hline Normal PSO & & 7.0399 & 46.621 \\
DPEEO & $(\mathrm{N}=22, \mathrm{M}=24)$ & 7.0402 & 3.751 \\
IDPEEO & & $\mathbf{7 . 0 4 0 4}$ & $\mathbf{1 . 1 1 8}$ \\
\hline Normal PSO & & 7.3811 & 104.877 \\
DPEEO & $(\mathrm{N}=30, \mathrm{M}=32)$ & 7.3809 & 5.884 \\
IDPEEO & & $\mathbf{7 . 3 8 1 2}$ & $\mathbf{1 . 3 7 3}$ \\
\hline
\end{tabular}

with mean $\varrho \tau$ and standard deviation $\sqrt{D_{\varrho} \tau}$ the nano-sensor's new position can be estimated. Furthermore, the parameters are assigned the values, such as maximum transmit power $P^{\max }=80 \mathrm{pJ}$, frequency width $\Delta f=0.1 \mathrm{THz}$, capacity of the power block is $200 p J$, and receive power $p_{r}^{t}=1 p J$.

2) Channel property of terahertz band: Figure 4 (a) and figure 4 (b) sequentially plot the total path loss and noise of the terahertz channel model versus the transmission distance and the frequency. To investigate the channel property of terahertz band, the transmission distance between nano-sensor and the sink node varies in the range from $0.5 \mathrm{~mm}$ to $4 \mathrm{~mm}$, and the frequency of the entire terahertz band from $0.5 \mathrm{THz}$ to $8 \mathrm{THz}$ is utilized. In Fig. 4, we observe that the Z-axis values are raised with the increase of the frequency and/or the transmission distance, especially for the path loss. It indicates that the distance and the frequency on communication path are dominant for the properties of the terahertz band.

3)Performance of the proposed algorithms: To verify the effectiveness of our algorithms, we compare our algorithms with a normal PSO. Table I shows the performance of the proposed algorithms over different network sizes. Compared with both the normal PSO and the DPEEO algorithm, the improved DPEEO algorithm can achieve a satisfactory EE within a shorter time. From Table I, we also observe that the $\mathrm{EE}$ and the convergence time both gradually increase with the network size.

Figure 5 plots the updates of the nano-sensors' transmit power strategies for one time slot by using the improved DPEEO algorithm. From figure 5, we see that the nano-sensor with high energy level will undertake more transmission tasks, owing to that our algorithm also considers the different energy states for various nano-sensors when executing resource allocation. Moreover, figure 5 not just shows the influences of energy resource on the nano-sensors' power controls, it also indicates the convergence of our algorithm. Figure 6 exhibits 


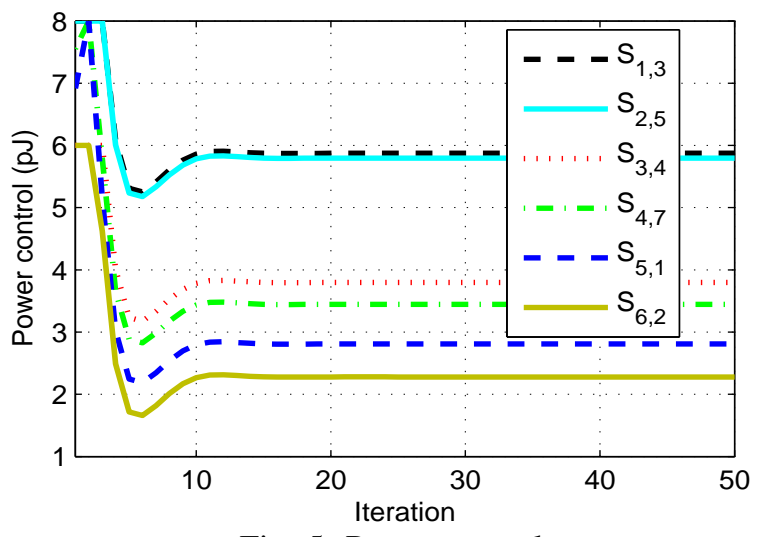

Fig. 5: Power control.

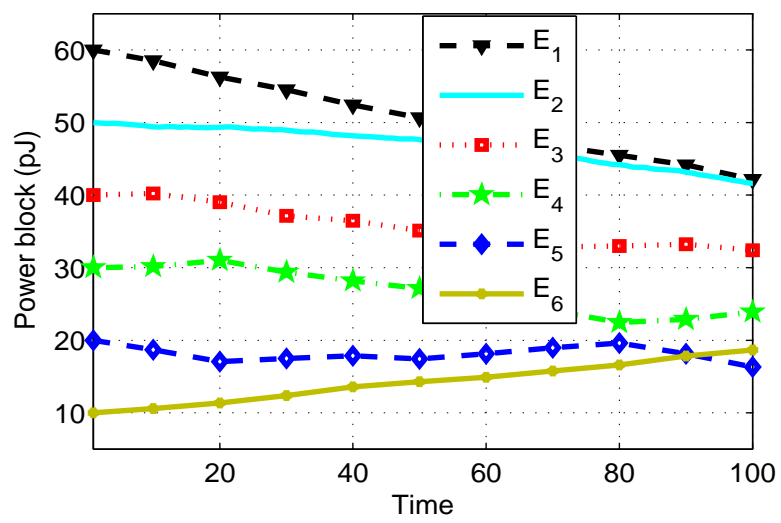

Fig. 6: Dynamic energy resource over time.

the dynamic power block process for nano-sensors over time. In figure 6, we can see that the gaps of the power blocks between different nano-sensors gradually decrease with time owing to that the nano-sensor with high energy level transmits more data packets, which can contribute to the energy balance among the nano-sensors in the networks. Figure 7 shows the achieved EEs versus the circuit power $P C$ under the improved DPEEO algorithm. From figure 7, we observe that when the circuit power increases, the EE of the algorithm reduces. That is because the channel capacity of network are independent of the circuit powers of nano-sensors, so that larger circuit power always leads to the smaller EE. Moreover, the initial positions

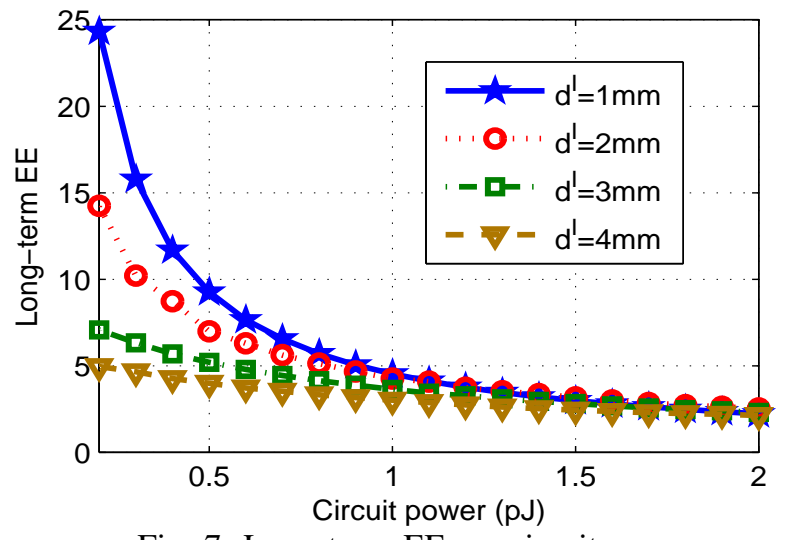

Fig. 7: Long-term EE vs. circuit power. of nano-sensors also have a influence on $\mathrm{EE}$, and the smaller initial positions will result in a larger EE for the network due to relative low path loss and noise.

\section{CONCLUSIONS}

In this paper, we introduced Brownian motion to describe the mobility of nano-sensors, and to built a time-variant terahertz channel model. Then, a long-term EE optimization problem is developed by virtue of the built channel model and the dynamic energy resources of nano-sensors. Based on equivalent transformation method, the non-convex optimization problem is then converted into a series of energyefficient resource allocation problems over the time slots. By employing the PSO method to solve the resource allocation problem, a DPEEO algorithm is devised to obtain a suboptimal solution for the EE optimization problem. Thus, by exploiting the special structure of the reformulated problems, an improved DPEEO algorithm is presented, which performed well in handling the problem's constraints and reducing the length of the convergence time.

\section{REFERENCES}

[1] L. Zhu, F.R. Yu, Y. Wang, B. Ning, and T. Tang, "Big data analytics in intelligent transportation systems: A survey,"' IEEE Transactions on Intelligent Transportation Systems, vol. 20, no. 1, pp.383-398, 2018.

[2] K. Yang, A. Pellegrini, M. Munoz and A. Brizzi, "Numerical Analysis and Characterization of $\mathrm{THz}$ Propagation Channel for Body-Centric Nano-Communications," IEEE Transactions on Terahertz Science and Technology, vol. 5, no. 3, pp. 419-426, May 2015.

[3] $\mathrm{M} . \mathrm{Ku}$ and J. Lai, "Joint Beamforming and Resource Allocation for Wireless-Powered Device-to-Device Communications in Cellular Networks," IEEE Trans. Wireless Communi., vol. 16, no. 11, pp. 7290-7304, Nov. 2017.

[4] G. Ramarao and K. Chandrasekaran, "Evaluating Lightning ChannelBase-Current Function Parameters for Identifying Interdependence of Wavefront and Tail by PSO Method," IEEE Transactions on Electromagnetic Compatibility, vol. PP, no. 99, pp. 1-8, June 2018.

[5] K. Singh, A. Gupta and T. Ratnarajah, "Green Resource Allocation and EE-balancing in Multiuser Two-way Amplify-and-Forward Relay Networks," in proc. of IEEE ICC, pp. 1-6, May 2017.

[6] M. Peng, Y. Yu, H. Xiang and H. Vincent Poor, "Energy-Efficient Resource Allocation Optimization for Multimedia Heterogeneous Cloud Radio Access Networks," IEEE Transactions on Multimedia, vol. 18, no. 5, pp. 879-892, May 2016.

[7] A. Afsharinejad, A. Davy and B. Jennings, "GA-based Frequency Selection Strategies for Graphene-based Nano-communication Networks,' Proc. of IEEE ICC, pp. 3642-3647, June 2014.

[8] J.Jornet and I. Akyildiz,"Channel Modeling and Capacity Analysis for Electromagnetic Wireless Nanonetworks in the Terahertz Band," Trans. Wireless Commun., vol.10, no.10, pp.3211-3221, Oct. 2011.

[9] G.Piro, G. Boggia and L.Grieco, "On the Design of an Energy-harvesting Protocol Stack for Body Area Nano-networks," Nano Communication Networks, vol.6, no.2, pp. 74-84, June 2015.

[10] E. Zarepour, M. Hassan, C. Chou and A. Adesina, "Frequency Hopping Strategies for Improving Terahertz Sensor Network Performance over Composition Varying Channels," Proc. of IEEE WoWMoM, pp. 1-9, June 2014.

[11] M. Kuscu and O. Akan, "A Communication Theoretical Analysis of FRET-Based Mobile Ad Hoc Molecular Nanonetworks," IEEE Trans. Nanobiosci., vol.13, no.3, pp.255-266, June 2014.

[12] J. Yang, Q. Yang, K. Kwak and R. Rao, "Power-Delay Tradeoff in Wireless Powered Communication Network," IEEE Trans. Veh. Technol., vol. 66, no. 4, pp. 3280-3292, Apr. 2017.

[13] C. Sung, M. Egan, Z. Chen and I. Collings, "Performance of Wireless Nano-Sensor Networks with Energy Harvesting," in proc. of IEEE VTC, pp. 1-5, Jan. 2015.

[14] J. Jornet, "A Joint Energy Harvesting and Consumption Model for SelfPowered Nano-Devices in Nanonetworks," in proc. of IEEE ICC, pp. 6151-6156, June 2012. 
[15] X. Wu and J. Lu, "Deterministic Coordinate Descent Algorithms for Smooth Convex Optimization," in proc. of IEEE CDC, pp. 709-714, Dec. 2017.

[16] M. Pourabdollah, B. Egardt, N. Murgovski and A. Grauers, "Convex Optimization Methods for Powertrain Sizing of Electrified Vehicles by Using Different Levels of Modeling Details," IEEE Trans. Veh. Technol., vol. 67, no. 3, pp. 1881-1893, Mar. 2018.

[17] N. Mokari, F. Alavi, S. Parsaeefard and T. Ngoc, "Limited-Feedback Resource Allocation in Heterogeneous Cellular Networks," IEEE Trans. Veh. Technol., vol. 65, no. 5, pp. 2509-2521, May 2015.

[18] D. Zhai, R. Zhang, L. Cai and B. Li, "Energy-Efficient User Scheduling and Power Allocation for NOMA-Based Wireless Networks With Massive IoT Devices," IEEE Internet of Things Journal, vol. 5, no. 3, pp. 1857 1868, Mar. 2018

[19] Z. Liu, H. Wei, X. Li and K. Liu , "Global Identification of Electrical and Mechanical Parameters in PMSM Drive based on Dynamic Self-Learning PSO," IEEE Transactions on Power Electronics, vol. PP, no. 99, pp. 1-13, Feb. 2018.

[20] A. Majid and E. Joelianto, "Optimal Sensor Deployment in Non-convex Region Using Discrete Particle Swarm Optimization Algorithm," in proc. of IEEE ICC, pp. 109-113, Feb. 2013.

[21] J. Jornet and I. Akyildiz, "Graphene-Based Nano-Antennas for Electromagnetic Nanocommunications in the Terahertz Band," in proc. of IEEE EuCAP, pp. 1227-1240, Apr. 2010.

[22] A. Singhal, R. Mallik and B. Lall, "Effect of Molecular Noise in Diffusion-Based Molecular Communication," IEEE Wireless Communications Letters, vol. 3, no. 5, pp. 489-492, Oct. 2014.

[23] K. Yang, A. Pellegrini, M. Munoz and A. Brizzi, "Numerical Analysis and Characterization of $\mathrm{THz}$ Propagation Channel for Body-Centric Nano-Communications," IEEE Trans. Terahertz Science and Technology, vol.5, no.3, pp.419-426, Apr. 2015.

[24] L. Wang, F. Hu, Z. Ling and B. Wang, "Wireless Information and Power Transfer to Maximize Information Throughput in WBAN," IEEE Internet of Things Journal, vol. 4, no. 5, pp. 1663-1670, Oct. 2017.

[25] J. Kwan and A. Fapojuwo, "Radio Frequency Energy Harvesting and Data Rate Optimization in Wireless Information and Power Transfer Sensor Networks," IEEE Sensors Journal, vol. 17, no. 15, pp. 4862-4874, June 2017.

[26] J. Jornet , "A Joint Energy Harvesting and Consumption Model for Self-Powered Nano-Devices in Nanonetworks," in proc. of IEEE ICC, pp. 6151-6156, June 2012.

[27] W. Wu, Q. Yang, P. Gong and K. Kwak, "Energy-Efficient Resource Optimization for OFDMA-Based Multi-homing Heterogenous Wireless Networks," IEEE Transactions on Signal Processing, vol. 64, no. 22, pp. 5901-5913, Nov. 2016

[28] S. Qiu, W. Haselmayr, B. Li and C. Zhao, "Bacterial Relay for EnergyEfficient Molecular Communications," IEEE Transactions on NanoBioscience, vol. 16, no. 7, pp. 555-562, Oct. 2017.

[29] C. Papadimitriou and K. Steiglitz, "Combinatorial optimization: algorithms and complexity," Prentice-Hall, 1982.

[30] C. He, B. Sheng, P. Zhu and X. You, "Energy-and Spectral-Efficiency Tradeoff for Distributed Antenna Systems with Proportional Fairness," IEEE Journal on Selected Areas in Communications, vol. 31, no. 5, pp. 894-902, May 2013.

[31] M. Kang, Y. Won, B. Lim and K. Kim, "Efficient Synthesis of Antenna Pattern Using Improved PSO for Spaceborne SAR Performance and Imaging in Presence of Element Failure," IEEE Sensors Journal, vol. 18 , no. 16 , pp. 6576-6587, June 2018.

[32] D. Sen, "The Uncertainty Relations in Quantum Mechanics," Current Science, vol. 107, no. 2, pp. 203C218, July 2014.

[33] Y. Zhao, V. Leung, X. Sun and Z. Chen, "Energy-Efficient Resource Allocation in Cellular Network with Ambient RF Energy Harvesting," in proc. of IEEE WCNC, pp. 1-6, May 2017.

[34] D. Tuong, Le. L and L. Tho, "Dual Decomposition Method for EnergyEfficient Resource Allocation in D2D Communications Underlying Cellular Networks," Proc. of IEEE GLOBECOM, pp.1-6, Dec. 2015.

[35] S. Boyd and L. Vandenberghe, "Convex optimization," Cambridge, U.K.:Cambridge Univ. Press, Mar. 2004.

[36] S. Koziel and Z. Michalewicz, "Evolutionary Algorithms, Homomorphous Mappings, and Constrained Parameter Optimization," Evolutionary Computation, vol. 7, no. 1, pp. 19C44, Mar. 1999.

[37] A. Ahmadzadeh, V. Jamali and R. Schober, "Stochastic Channel Modeling for Diffusive Mobile Molecular Communication Systems," IEEE Trans. Commun., vol. PP, no. 99, pp. 1-17, July 2018.

[38] L. Feng, K.S. Kwak, and Q. Yang, "October. Resource allocation for time-variant channels in the nano-communication networks," In Proc. IEEE International Conference on Information and Communication Technology Convergence (ICTC), 2018, pp. 667-671. 